\title{
The reticular atomic filter in the vacuum. The adaptability of the electronic cloud of atoms with two applications: hydrogen and water.
}

\author{
Authoress: Giovanna D'Alonzo \\ Degree in Astronomy, University of Bologna, Bologna, Italy \\ e-mail: giovanna.dalonzo82@gmail.com
}

\begin{abstract}
In the vacuum, some solids could be used as gas filters not only through the empty spaces of the crystal lattice but also through the electronic clouds of the atoms that make up the solid. With the use of particular equipment in the vacuum we could obtain a new energy, look for the energy sufficient for the adaptability of the electronic cloud and obtain a suitable temperature for the adaptability of the electronic cloud. In the case suppose that the electronic clouds of the atoms constituting the solid are valued by the ions, this energy is subtracted from ion ionization energy in the total energy of the molecules distribution and, through some mathematical passages, we deduce a temperature sufficient for the passageway of the electronic cloud suitable for some solids. An ion and/or an atom to be filtered should have a smaller diameter than the atomic radius of the atom that constitutes the filter, for the transition through the electronic clouds. The gases could cross the solid or in the empty spaces of the crystal lattice or in the electronic clouds of the atoms that make up the solid due to the difference between the force fields. Possible future applications could improve the quality of life, in particular it would be possible to reduce atmospheric pollution by producing molecular hydrogen which would become above all a solid ecological alternative for energy production and, therefore, a solid alternative to nuclear power.
\end{abstract}

keywords: adaptability of the electronic cloud, molecular hydrogen 


\section{Introduction}

This research is the result of a scientific intuition concerning the hypothesis, still to be tested, of the adaptability of the electronic cloud of atoms which constitute a solid thickness from the nanometer up to a few micrometers by some neutral atoms and/or ions and/or molecules and/or electrons by means of special instruments in the vacuum. An atom can be traversed in certain conditions by something like the atom itself with internal energy, namely: another atom, an ion, an electron and/or a molecule. The energies and temperatures I consider make sure that during the transit the atom, the ion and/or the molecule do not incorporate the nuclei of the atoms constituting the solid with their electronic cloud. The nuclei of the atoms constituting the solid are as if they were the "pillars of the solid" and the transit would take place between the nucleus of the atom and the very surface of the atom constituting the solid. The atom that crosses the atom that constitutes the solid has less internal energy than the internal energy of the constituent atom of the solid. I suppose that the electron cloud of the crossing atom remains compact and that the electrons of the constituent atom of the solid are distributed in their remaining electronic cloud during the passage. The necessary pressure is an index that reveals us under what conditions this result can be reached, in addition to temperature, to the increase of the power of natural kinetic energy, to the harmonic ascillations of the solid, etc. Therefore, an atom or an ion valiant the electronic cloud must have an atomic or ionic diameter smaller than the atomic radius of the atom constituting the solid. Given the energies and temperatures that I consider, the atom, the ion, the electron and/or the molecule would naturally affect the solid either in the empty gaps of the crystalline lattices or in the electronic clouds of the atoms that make up the solid, considering the different fields of forces. The theoretical results show that, starting from the molecular distribution equation with some mathematical steps, it is possible to obtain a suitable temperature for some solids, so that this result can be obtained. In fact, supposing that an ion can cross the electronic cloud of the atoms that make up the solid, in the distribution equation we could consider the total energy as the difference between the ionization energy, the gravity energy to which the ion is subject, and, by means of special instruments in the void that produce a new energy, we could subtract the new energy with a numerical variable $\mathrm{x}$ to be found. Assuming the total constant energy setting a suitable temperature for the solid, through the distribution of the molecules, we could obtain a differential logarithmic equation and with its integration we could obtain the numerical variable that verifies the equation and is applied at a temperature suitable for some solid. 
On the contrary, supposing that a neutral atom can cross the electronic cloud of the atoms that make up the solid, due to the absence of a positive energy like ionization energy for the ions, the logarithmic equation would not be defined. However, from the physical point of view, it can be assumed that given the absence of the "obstacle" of ionization energy for the neutral atom, this result could be achieved. Moreover, an ion and/or an atom to be filtered should have a smaller diameter than the atomic radius of the atom that constitutes the filter, for the passage through the electronic clouds. lons, electrons, neutral atoms and molecules could cross the solid or in the empty spaces of the crystal lattice or in the electronic clouds of the atoms that make up the solid because of the difference between the force fields. Indeed, Coulomb's forces in the empty spaces of the crystal lattice are smaller than Coulomb's forces in electronic clouds. The atom and/or ion would naturally check the solid in one of the two fields of forces that are convenient to them, and during filtration through the electronic clouds they would cross the atoms in the space between the nucleus and the surface of the atom itself without incorporating the nuclei of the atoms that make up the solid, in order to avoid some potential and force fields among which the Yukawa potentials that would be high for this project. The nuclei of the atoms that make up the solid would become the "pillars of the solid" while in the solid surface the filtration would occur in two different force fields, with low filtration energy and at suitable temperatures for some solids. Filtration would be stable and highly selective. The solid I consider is black phosphorus with its solvation shell for a highly selective filtration.

\section{Materials and methods}

\section{Because an atom or an ion should cross the electronic cloud of another suitable atom}

A set of particles No at temperature $\mathrm{T}$ is arranged according to the curve:

$$
(1 / N o) N(E)=\frac{2}{\sqrt{\pi} \cdot k^{(3 / 2)}} \cdot\left(E^{(1 / 2)} /\left(T^{(3 / 2)}\right)\right) \cdot e^{(-E / k T)}
$$

where $N(E)$ is the number of particles for energy and the average energy is:

$$
\frac{1}{N o} \cdot \int E \cdot N(E) d E=3 / 2 k T
$$

In the previous formulas we can consider $\mathrm{E}$ as a constant, setting the temperature (eg $\mathrm{T}=$ $396.28 \mathrm{~K}$ ) and considering $\mathrm{E}$ as the ionization energy (constant), if it is an ion, plus the potential gravitational energy (constant) to which the ion or atom is subject, in addition to a new energy due to special instruments that could be void fans $V=-(3 / 2) \cdot k \cdot T \cdot x \quad$ with a 
numeric variable to be found. That is, we pull out of the integral $E$ and $N(E)$, replace $(1 / \mathrm{No})$ $N(E)$ with the first formula and, finally, the integral $\int d E=E$. Therefore, we would get:

- (3) $\quad T^{(5 / 2)} \cdot e^{(E / k T)}=\frac{4}{3 \cdot \sqrt{\pi} \cdot k^{(5 / 2)}} \cdot E^{(5 / 2)}$

Assuming $\quad \frac{4}{3 \cdot \sqrt{\pi} \cdot \mathrm{k}^{(5 / 2)}} \mathrm{E}^{(5 / 2)}=\mathrm{cost} \quad$ and applying the logarithms:

- (4) $\quad(5 / 2) \ln (\mathrm{T})+\mathrm{E} / \mathrm{kT}=\ln ($ cost $)$

We apply the following substitutions:

- $y=\ln (T)$

- $y^{\prime}=1 / T$

and the following initial conditions:

- $\mathrm{y}(1)=0$

Thus, the equation becomes a differential equation:

- $\quad(E / k) y^{\prime}(T)+(5 / 2) y(T)=\log ($ cost $)$

Then

- $y(T)=\mathrm{e}^{-\left(\frac{5}{2}(\mathrm{~T}-1)\right)} \cdot\left(0+\int_{1}^{\mathrm{T}}\left(\ln (\cos \mathrm{t}) \cdot\left(\mathrm{e}^{\frac{5}{2}(\mathrm{~T}-1)}\right) \mathrm{dT}\right)\right)=\mathrm{e}^{-\left(\frac{5}{2}(\mathrm{~T}-1)\right)} \ln (\cos \mathrm{t}) \cdot\left(\frac{2}{5}\right) \cdot\left(\mathrm{e}^{\left(\frac{5}{2}(\mathrm{~T}-1)\right)}-1\right)$

- $y(T)=\left(\frac{2}{5} \ln (\cos t)\right)-\left(\frac{2}{5} \ln (\cos t) \mathrm{e}^{-\left(\frac{5}{2}(\mathrm{~T}-1)\right)}\right)$

Then

- $\mathrm{y}(\mathrm{T})=\frac{2}{5} \cdot \ln ($ cost $) \cdot\left(1-\mathrm{e}^{-\left(\frac{5}{2}(\mathrm{~T}-1)\right)}\right)$

where is it $\quad \mathrm{e}^{-\left(\frac{5}{2}(\mathrm{~T}-1)\right)} \sim 0$

Then

- (5)

$$
\mathrm{y}(\mathrm{T}) \sim \frac{2}{5} \cdot \ln (\text { cost })
$$

An atom can be traversed in certain conditions by something like the atom itself with internal energy, namely: another atom, an ion, an electron and/or a molecule. The energies and temperatures I consider make sure that during the transit the atom, the ion and/or the molecule do not incorporate the nuclei of the atoms constituting the solid with their electronic cloud. The nuclei of the atoms constituting the solid are as if they were the "pillars of the solid" and the transit would take place between the nucleus of the atom and the very surface of the atom constituting the solid. Therefore, an atom or an ion valiant the electronic cloud must have an atomic or ionic diameter smaller than the atomic radius of the atom constituting the solid. Given the energies and temperatures that I consider, the atom, the ion, the electron and/or the molecule would naturally affect the solid either in the empty gaps of the crystalline 
lattices or in the electronic clouds of the atoms that make up the solid, considering the different fields of forces. Considering a new energy that takes into account the new energy caused by the special instruments that would direct the atoms and ions to the filter and increase the power of the kinetic energy of atoms and ions, namely:

$$
\frac{3}{2} \cdot k T \cdot x
$$

we could subtract this energy from the ionization energy in the $E$ of the Boltzmann equation, since, most likely, many atoms and/or ions would have sufficient energy for the viability of the electronic cloud and would be directed to the filter, ie:

$$
\text { - (6) } \quad E=\text { Eionization - Egravitypotential }-\frac{3}{2} \cdot k T \cdot x
$$

By setting an acceptable temperature value (for example $T=396.28 \mathrm{~K}$ ), we must find a numerical variable $x$ able to satisfy the equation:

- $\mathrm{y}(\mathrm{T}) \sim \frac{2}{5} \cdot \ln (\cos \mathrm{t})$

The atom or ion to be filtered should have an atomic diameter smaller than the atomic rays of the atoms that make up the solid so that the filtration through the electronic clouds takes place in the space between the nuclei and surfaces of the atoms that make up the solid. During the filtration through the electronic clouds, the distance between the nuclei of the atom constituting the solid and of the atom or the ion that crosses the electronic cloud is on average some tens of picometers, larger than the distance at which there are considerable effects of Yukawa potential, as shown in the figure:

1)

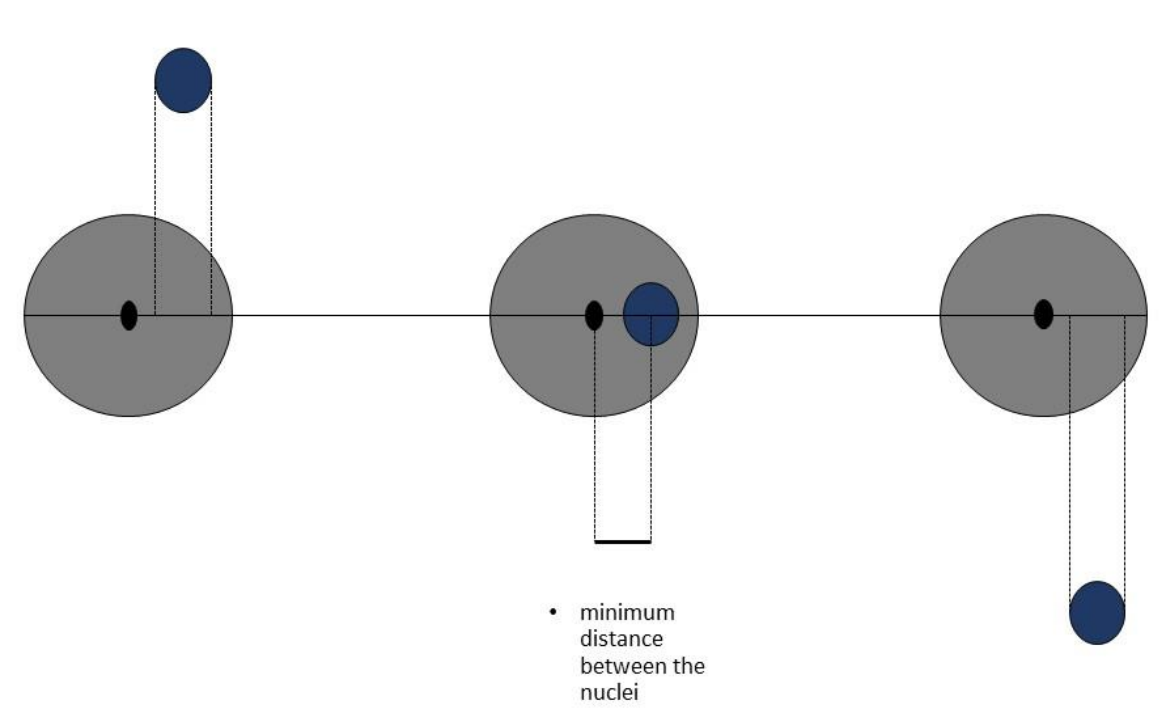


suitable for some solids, or in the empty spaces of the crystal lattice or in the electronic clouds of the atoms that make up the solid but not both because of the difference of force fields. In this way the electronic clouds of atoms or ions they do not incorporate the nuclei of the atoms that make up the solid in the filtration through electronic clouds in order to avoid some fields of forces and potentials including the Yukawa Potential that is high for this project. In fact, the minimum distance between the nuclei of the atom that constitutes the solid and of the atom or the ion that crosses the solid in the field of electronic cloud forces is on average some tens of picometers and this minimum distance is greater than the distances at what are the considerable effects of the Yukawa potential. Moreover, the electronic cloud of the atom that crosses the solid in the field of forces of the electronic cloud of the atom that constitutes the solid does not incorporate the nucleus of the atom constituting the solid during its passage, given the low energies and temperatures considered. In this way the nuclei of the atoms that compose the solids would become the "pillars of the solid" making the filtration stable and effective and above all selective. Filtration would occur at low energies and at low temperatures suitable for some solids. In fact, between the nuclei and the surfaces of the atoms that make up the filter there is a field of forces greater than that in the empty spaces of the crystalline lattice of the solid. The solid should filter electrons and/or ions and/or molecules and/or neutral atoms, depending on the circumstances. The latter could cross the solid or in one field or the other, ie in the electronic clouds or in the empty spaces of the lattice due to the difference of the force fields. In fact, to allow filtering, the Coulomb forces to be overcome will be smaller in the empty spaces of the crystalline lattice of the solid compared to the Coulomb forces to be overcome for the passage in the electronic cloud. As for a neutral atom, from this formula

- $\mathrm{y}(\mathrm{T}) \sim \frac{2}{5} \cdot \ln ($ cost $) \quad$ where is it $\frac{4}{3 \cdot \sqrt{\pi} \cdot \mathrm{k}^{(5 / 2)}} \mathrm{E}^{(5 / 2)}=$ cost

$$
\mathrm{E}=\text { Eionization }- \text { Egravitypotential }-\left(\frac{3}{2} \cdot \mathrm{kT}\right) \cdot \mathrm{x}
$$

it is clear that we would obtain a negative energy, since we would not consider ionization energy, and therefore the logarithm would not be defined. Therefore, nothing can be said about the adaptability of the electronic cloud by a neutral atom. However, from the physical point of view, it is plausible that even the neutral atom can cross the electronic cloud in the absence of the "obstacle" of ionization energy. 


\section{Molecular hydrogen}

The ionization energy of the hydrogen atom is:

- (7) $\quad \mathrm{Ei}=+13,6 \mathrm{eV}=+13,6 \cdot 1,602176565 \cdot 10^{-19} \mathrm{~J}=+2,178960128 \cdot 10^{-18} \mathrm{~J}$

The gravitational potential energy is:

- (8)

$$
\mathrm{U}=\frac{-\mathrm{G} \cdot \mathrm{M} \cdot \mathrm{m}}{\mathrm{r}} \sim-1,047132023 \cdot 10^{-19} \mathrm{~J}
$$

where $M$ is the mass of the Earth, $m$ is the mass of the hydrogen atom and $r$ is the terrestrial ray. The kinetic energy is the following: $K=3 / 2 \cdot k \cdot T$ where $k$ is the Boltzmann constant. By setting an acceptable temperature of $T \sim 396.28 \mathrm{~K}$ and assuming to supply new energy through special instruments that would increase the power of kinetic energy and which would direct atoms and/or ions and/or electrons to the filter, the latter they would have sufficient energy to make the electronic cloud suitable, and this new energy could be written as follows:

$$
\mathrm{V}=-\left(\frac{3}{2}\right) \cdot \mathrm{k} \cdot \mathrm{T} \cdot \mathrm{x}
$$

where $\mathrm{x}$ is a numeric variable to find.

Being $\quad \mathrm{y}(\mathrm{T}) \sim \frac{2}{5} \ln (\cos \mathrm{t}) \quad$ where is it $\quad \mathrm{y}(\mathrm{T})=\ln (\mathrm{T}) ; \cos \mathrm{t}=\frac{4}{3 \sqrt{\pi}} \cdot\left(\frac{\mathrm{E}}{\mathrm{k}}\right)\left(\frac{5}{2}\right)$;

$$
\mathrm{E}=\text { Eionization }- \text { Egravitypotential }-\frac{3}{2} \cdot \mathrm{kT} \cdot \mathrm{x}
$$

after assuming the temperature of $396.28 \mathrm{~K}$, we must find a numeric variable $\mathrm{x}$ that satisfies the equation:

- (9) $\quad T \sim 396,28 K$

$$
x \sim 252
$$

Therefore, it is necessary to increase the power of the natural kinetic energy $V=-(3 / 2) \cdot K \cdot T \cdot x$ with $x \sim 252$ in the vacuum, bringing the solid to a temperature of $396.28 \mathrm{~K}$. 


\section{Results}

\section{The instrumentation for molecular hydrogen}

One possible application could be to oxidize water in a vacuum in a glass or steel container by means of a cobalt ${ }^{1}$ and ultraviolet light catalyst. The filter should be a thick solid from the nanometer to a few micrometers of black phosphorus ${ }^{2}$, since the black phosphorus has a solvent shell and therefore the solid would only filter the hydrogen ions and the electrons. The necessary equipment would generally be the following: in a vacuum, a steel or glass container for molecular oxygen, $\mathrm{H}+$ ions and electrons; the upper surface of the container should be a led piston which in addition to overcoming the Coulomb forces in the crystal lattice would have two other tasks, induce water oxidation and prevent the recombination of ions and hydrogen electrons before filtration; special tools such as fans should be incorporated into the piston; they should be able to produce an energy that could contribute, through the distribution of molecules and through mathematical passages, to an adequate temperature; the steel or glass container must have a removable bottom; the solid should oscillate harmoniously by some micrometers to facilitate filtration; an instrument capable of heating the solid at a temperature of $396.28 \mathrm{~K}$; two thin metal grids to support the solid; the solid should be placed on an instrument capable of sucking $\mathrm{H}+$ ions and electrons which will be "filtered" by the solid and collected in a steel container; the suction tool should take more time in the direct direction and a shorter time to aspirate in the opposite direction to avoid the obstruction of the solid mainly in the empty spaces of the crystal lattice and also the piston should move some micrometers from the bottom to the top in the the very moment in which the aspiration takes place in the opposite direction with the same instrument to avoid the obstruction of the empty spaces and the breaking of the solid. Moreover, in the electronic clouds of the atoms constituting the solid, there is a field of forces greater than that in the empty spaces of the solid. For more efficient filtration, the solvation shell should protect the black phosphorus only in the upper part, which would be in contact with molecular oxygen and in direct contact with the pressure force exerted by the piston. From the quantum-mechanical equations for electronic states, it can be deduced that few Newtons of piston pressure force are sufficient for such a project. The instrumental equipment is as follows: 


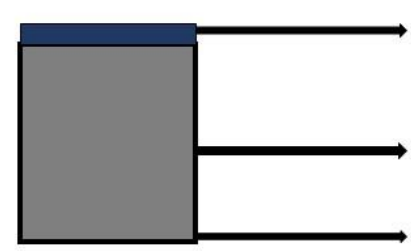

LED piston with fans

Container for the oxidation of water

Removable bottom

Black phosphorus with support grids and harmonic oscillations

Tool to heat the solid to the appropriate temperature

Instrument to aspirate the hydrogen ions and electrons

Molecular hydrogen container

So far we have used pure water, but we could also use impure water or sea water for this hypothetical filtration. In the table below, we have reported the main atomic or ionic rays of the respective elements or ions present in marine waters ${ }^{3}$.

\begin{tabular}{|c|c|c|c|c|c|c|c|}
\hline Element & $\begin{array}{l}\text { Atomic } \\
\text { Number }\end{array}$ & $\begin{array}{l}\text { Ionic or } \\
\text { atomic } \\
\text { radius }\end{array}$ & $\begin{array}{l}\text { Atomic } \\
\text { weight }\end{array}$ & Element & $\begin{array}{l}\text { Atomic } \\
\text { number }\end{array}$ & $\begin{array}{l}\text { Ionic or } \\
\text { atomic } \\
\text { radius }\end{array}$ & $\begin{array}{l}\text { Atomic } \\
\text { weight }\end{array}$ \\
\hline $\mathrm{Cl}$ & 17 & $181 \mathrm{pm}$ & $35,5 \mathrm{u}$ & $u$ & 92 & $52 \mathrm{pm}$ & $238 \mathrm{u}$ \\
\hline $\mathrm{Na}$ & 11 & $102 \mathrm{pm}$ & $22,9 \mathrm{u}$ & $\mathrm{Ni}$ & 28 & $69 \mathrm{pm}$ & $58,7 \mathrm{u}$ \\
\hline$M g$ & 12 & $72 \mathrm{pm}$ & $24,3 \mathrm{u}$ & $v$ & 23 & $135 \mathrm{pm}$ & $50,9 \mathrm{u}$ \\
\hline$S$ & 16 & $37 \mathrm{pm}$ & $32 \mathrm{u}$ & $M n$ & 25 & $46 \mathrm{pm}$ & $54,9 \mathrm{u}$ \\
\hline $\mathrm{Ca}$ & 20 & $100 \mathrm{pm}$ & $40 \mathrm{u}$ & $\pi i$ & 22 & $61 \mathrm{pm}$ & $47,8 \mathrm{u}$ \\
\hline$K$ & 19 & $138 \mathrm{pm}$ & $39 \mathrm{u}$ & $s b$ & 51 & $76 \mathrm{pm}$ & $121,7 \mathrm{u}$ \\
\hline$B r$ & 35 & $196 \mathrm{pm}$ & $90 \mathrm{u}$ & Co & 27 & $75 \mathrm{pm}$ & 58,9 u \\
\hline C & 6 & $70 \mathrm{pm}$ & $12 \mathrm{u}$ & $\mathrm{Ce}$ & 58 & $103,4 \mathrm{pm}$ & $140 \mathrm{u}$ \\
\hline$S r$ & 38 & $112 \mathrm{pm}$ & $88 \mathrm{u}$ & $Y$ & 39 & $90 \mathrm{pm}$ & 88,9 u \\
\hline$B$ & 5 & $23 \mathrm{pm}$ & $10,8 \mathrm{u}$ & $A g$ & 47 & $126 \mathrm{pm}$ & $107,9 \mathrm{u}$ \\
\hline si & 14 & $40 \mathrm{pm}$ & $28 \mathrm{u}$ & $L a$ & 57 & $106 \mathrm{pm}$ & $138,9 \mathrm{u}$ \\
\hline$F$ & 9 & $133 \mathrm{pm}$ & $19 \mathrm{u}$ & Cripton & 36 & $88 \mathrm{pm}$ & $83,8 \mathrm{u}$ \\
\hline Argon & 13 & $71 \mathrm{pm}$ & $40 u$ & Neon & 10 & $38 \mathrm{pm}$ & $20 \mathrm{u}$ \\
\hline$N$ & 7 & $13 \mathrm{pm}$ & $14 \mathrm{u}$ & $C d$ & 48 & $97 \mathrm{pm}$ & $112,5 \mathrm{u}$ \\
\hline$L i$ & 3 & $76 \mathrm{pm}$ & $7 \mathrm{u}$ & $w$ & 74 & $62 \mathrm{pm}$ & $183,8 \mathrm{u}$ \\
\hline$R b$ & 37 & $152 \mathrm{pm}$ & $85,5 \mathrm{u}$ & Xeno & 54 & $108 \mathrm{pm}$ & $131,3 \mathrm{u}$ \\
\hline$S n$ & 50 & $69 \mathrm{pm}$ & $118,7 \mathrm{u}$ & Ge & 32 & $53 \mathrm{pm}$ & $72,6 \mathrm{u}$ \\
\hline$P$ & 15 & $38 \mathrm{pm}$ & $30,9 \mathrm{u}$ & $\mathrm{Cr}$ & 24 & $52 \mathrm{pm}$ & $52 \mathrm{u}$ \\
\hline 1 & 53 & $220 \mathrm{pm}$ & $126,9 \mathrm{u}$ & Th & 90 & $97,2 \mathrm{pm}$ & $232 \mathrm{u}$ \\
\hline$B a$ & 56 & $135 \mathrm{pm}$ & $137,3 \mathrm{u}$ & $S c$ & 21 & $75 \mathrm{pm}$ & $45 \mathrm{u}$ \\
\hline In & 49 & $80 \mathrm{pm}$ & 114,8 u & $P b$ & 82 & $119 \mathrm{pm}$ & $207 \mathrm{u}$ \\
\hline$Z n$ & 30 & $74 \mathrm{pm}$ & $65,4 \mathrm{u}$ & $\mathrm{Hg}$ & 80 & $102 \mathrm{pm}$ & $200,6 \mathrm{u}$ \\
\hline $\mathrm{Fe}$ & 26 & $140 \mathrm{pm}$ & $55,8 \mathrm{u}$ & $G a$ & 31 & $62 \mathrm{pm}$ & $69,7 \mathrm{u}$ \\
\hline$A l$ & 13 & $54 \mathrm{pm}$ & $26,9 u$ & $B i$ & 83 & $103 \mathrm{pm}$ & $208,9 \mathrm{u}$ \\
\hline Mo & 42 & $65 \mathrm{pm}$ & $95,9 \mathrm{u}$ & $N b$ & 41 & $69 \mathrm{pm}$ & $93 \mathrm{u}$ \\
\hline $\mathrm{Se}$ & 34 & $50 \mathrm{pm}$ & $78,9 \mathrm{u}$ & $\pi I$ & 81 & $150 \mathrm{pm}$ & $204,3 \mathrm{u}$ \\
\hline $\mathrm{Cu}$ & 29 & $73 \mathrm{pm}$ & $63,5 \mathrm{u}$ & Elio & 4 & $128 \mathrm{pm}$ & $4 \mathrm{u}$ \\
\hline As & 33 & $58 \mathrm{pm}$ & $74,9 \mathrm{u}$ & $A u$ & 79 & $85 \mathrm{pm}$ & $196,9 \mathrm{u}$ \\
\hline
\end{tabular}

Many of the ions, molecules, elements of heavy metals that make up ocean waters would result as residues in the bottom that would be extracted through a resealable side opening. Some gases, such as fluorine, argon, nitrogen, cripton, neon, xenon and helium, would persist; they have spatial dimensions greater than the dimensions of the empty spaces of 
the black phosphorus solvation shell; therefore, the shell would filter only the ions and the electrons of hydrogen. The force to be applied through the piston would remain the same as that previously calculated. After filtration, the hydrogen ions and the electrons would recombine to form molecular hydrogen due to the absence of ultraviolet light. Finally, by combining molecular hydrogen and molecular oxygen into a glass or steel container, good water could be obtained through a reduction reaction from impure water, including that of rivers, lakes, rainwater and the sea. In fact, most components of impure water were deposited on the removable bottom of the first container after water oxidation and, together with molecular oxygen, only a small percentage of the gas remained.

\section{Conclusion}

The methodology is applied in the search for a suitable temperature, using a distribution equation and a special vacuum equipment responsible for energizing the gas, assuming that the atoms and ions have an atomic or ionic diameter less than the atomic radius of the atoms. that make up the solid so that the atoms are able to cross the electronic cloud in one of the two force fields: in the empty spaces of the crystal lattice or in the electronic clouds. This method should allow the solid to become a filter on all its surface and the nucleus of the atoms that make up the solid should become the "pillars of the solid" in order to make the filtration stable and effective and avoid the breaking of the solid, and above all become selective. At present, there are various filtration techniques, particularly the molecular filter. Regarding the treatment of wastewater, scientists have studied molecular filters composed of bio-renewable materials ${ }^{4}$ and as for water desalination ${ }^{5}$ there are some particular techniques such as the use of graphene membranes ${ }^{6}$. Furthermore, hydrogen filtration is currently occurring on atomic solids, the hyperpure molecular hydrogen is produced by filtration on noble metals ${ }^{7}$. These researches are the result of a scientific intuition that, if the experimentation was successful, could improve the quality of life in many sectors, such as the environmental branch with the realization of molecular hydrogen in an alternative way. This synthesis of molecular hydrogen is significantly able to reduce air pollution caused by all types of fuels used in factories and by gasoline and gases used in vehicles. Molecular hydrogen is an environmentally friendly fuel and therefore does not cause damage to the environment and, over the decades, is significantly able, if well used in the world, to reduce the greenhouse effect and atmospheric pollution, contributing to decisive way to redefine the atmospheric balance of our planet. Furthermore, the molecular synthesis of hydrogen could also occur using impure water such as rain, sea or river water using the same 
procedure as regards pure or distilled water in order to prevent the reduction of water resources, case in which the experiments give positive results. Furthermore, by applying this scientific hypothesis to be experimented, water, the most precious commodity in the world, could be transformed into good water even in those places in the world where it is polluted, contributing undoubtedly to improving the quality of life and health of people. Furthermore, there are many possible applications for the good of the ecosystem. For example, starting from the principle 'nothing to create, nothing is destroyed everything is transformed' you can try to naturally transform one molecule into another through the reticular atomic filter and various techniques in a vacuum: for example, you can try to transform chlorophyll natural in natural cellulose for paper production. A similar technique could also be used for the production of plastic, etc. Furthermore, since the molecules produced are natural and are produced through various technical passages in a vacuum, substances could be naturally created by means of the lattice atomic filter biodegrade what has been achieved. Another future application of my research could be a valid aid in biological and medical research. Furthermore, the stabilization of visible and non-visible light could also be another possible future application. Finally, we could extend the concept of spatial fields from crystal lattices to macrocosm by reproducing metal structures supporting objects by reproducing the crystal lattices of these metals in proportion to space and weight to achieve greater stability.

\section{References}

(1) Miao Zhang et all. Time-resolved observations of water oxidation intermediates on a cobalt oxide nanoparticle catalyst. Nature chemistry 6, 362-367 (2014)

(2) Michele Buscema et all. Photovoltaic effect in few-layer black phosphorus PN junctions defined by local electrostatic gating. Nature Communications 5, Article number 4651(2014)

(3) Element, atomic number, ionic or atomic radius.

https://en.wikipedia.org/wiki/Atomic_radius

https://it.wikipedia.org/wiki/Raggio_ionico

(4) Asli Celebioglu et al. Electrospun crosslinked poly-cyclodextrin nanofibers: Highly efficient molecular filtration thru host-guest inclusion complexation. Scientific Reports 7, article 
number $7369(2017)$

(5) Bumjoo Kim et al. Purification of High Salinity Brine by Multi-Stage Ion Concentration Polarization Desalination.Scientific reports 6,article number: 31850 (2016)

(6) Albert Boretti et al. Outlook for graphene-based desalination membranes. npj Clean Water 1, Article number: 5 (2018)

(7) Palladium-Based Alloy Membranes for Separation of High Purity Hydrogen from HydrogenContaining Gas Mixtures

https://www.technology.matthey.com/pdf/3-12-pmr-jan11.pdf 